\title{
GENERATION OF ULTRA-SHORT RELATIVISTIC-ELECTRON-BUNCH BY A LASER WAKEFIELD*
}

\author{
A. G. Khachatryan, K.-J. Boller, and F. A. van Goor, \\ Laser Physics \& Nonlinear Optics Group, Dept. of Applied Physics, University of Twente, P.O. \\ Box 217, 7500 AE Enschede, The Netherlands
}

\begin{abstract}
The possibility of the generation of an ultra-short (about one micron long) relativistic (up to a few $\mathrm{GeVs}$ ) electron-bunch in a moderately nonlinear laser wakefield excited in an underdense plasma by an intense laser pulse is investigated. The ultra-short bunch is formed by trapping, effective compression (both in longitudinal and in transverse directions) and acceleration of an initially nonrelativistic (with kinetic energy of a few hundreds $\mathrm{keVs}$ ) e-bunch that is injected in front of the laser pulse. The initial bunch may be of poor quality, may have a duration in the order of the laser pulse length or longer and can be generated by a laser-driven photo-cathode RF gun. Our $1 \mathrm{D}$ and $3 \mathrm{D}$ calculations predict that the accelerated ultra-short bunch will show a low energy spread of less than one percent and a low transverse emittance in the order of a nanometer. An energy gain in the GeV-range is feasible at an accelerating distance of a few centimetres. The total number of accelerated electrons is restricted by the beam loading effect only and can reach a value of $10^{8}-10^{9}$.
\end{abstract}

\section{INTRODUCTION}

Electron bunches with the length in the order of a hundred microns have been produced using a photocathode radio-frequency (RF) gun [1], a thermionic RF gun [2], and a magnetic bunch compressor [3]. However, many applications, such as laser wake-field acceleration, inverse Cherenkov acceleration, inverse free electron laser (FEL), x-ray FEL, high energy physics and other applications, require much shorter electron bunches with a length in the order of a micrometer. Generation of such a short electron bunch is a difficult technical problem, new approaches to which are required. Recently, a new scheme of a laser wake-field accelerator (LWFA) has been proposed $[4,5]$, which allows the generation of an ultra-short, ultra-relativistic, high quality electron bunch. The scheme utilizes a non(weakly)-relativistic bunch of electrons which is injected in front of a high-intensity laser pulse generating a nonlinear wake wave in an underdense plasma (see Fig. 1). Our results [4,5] showed that the electron bunch runs through the pulse, is trapped in the first accelerating maximum in the wake, essentially compressed both in longitudinal and transverse directions, and accelerated to an ultra-relativistic energy: Here we

*This work is supported by Fundamenteel Onderzoek der Materie (FOM, The Netherlands) under "Laser Wakefield" project. show that the trapped electron bunch length is much less than the plasma wavelength. We also show that the generation of ultra-short relativistic bunches by our scheme is possible even when the initial bunch length is much longer than the plasma wavelength.

\section{ONE-DIMENSIONAL (1D) THEORY}

Because the trapped electrons are concentrated close to the axis, their longitudinal dynamics are well described by a $1 \mathrm{D}$ theory [5]. The $1 \mathrm{D}$ theory also allows a more detailed description of the problem. Suppose that an electron is initially ahead of the laser pulse (see Fig. 1) and moves with a velocity $v_{0}$ less than the group velocity

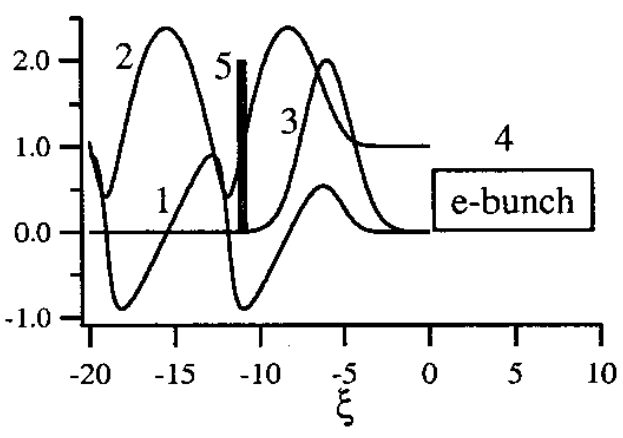

Figure 1: The laser wakefield scheme: the wake electric field $E_{z}(1)$, the potential $\Phi(2)$, the laser pulse amplitude $a(3)$, the initial (4) and trapped (5) bunches as a function of the position in a moving frame. $a_{0}=2, \sigma_{z}=2, \gamma_{s}=50$.

of the pulse $v_{g}$, which, in turn, is equal to the wake phase velocity. From the well known integral of motion $\gamma \beta_{g} p-\Phi=$ const (see, e.g., [4]; where $\gamma$ and $p$ are the gamma factor and the momentum of the electron normalized to $m_{e} c, \beta_{g}=v_{g} / c, \Phi(\xi)=1+e \varphi / m_{e} c^{2} \equiv 1+\phi$ is the normalized wake potential, $\xi=k_{p}\left(Z-v_{g} t\right) \cong z-\tau, k_{p}=\omega_{p} / v_{g}$, $\omega_{p}=\left(4 \pi n_{p} e^{2} / m_{e}\right)^{1 / 2}$ is the plasma frequency, and $n_{p}$ is the unperturbed plasma electron concentration) one can obtain an expression for the initial momentum of the trapped electron $[4,5]$ :

$$
p_{0}=\gamma_{g}^{2}\left[\beta_{g} S-\left(S^{2}-\gamma_{g}^{-2}\right)^{1 / 2}\right]
$$

where $S=1 / \gamma_{g}+1-\Phi_{t r}, \Phi_{t r}$ is the wake potential at the trapping point $\xi_{r r}$, at which $p=\beta_{g} \gamma_{g} \equiv p_{g}, \gamma_{g}=\left(1-\beta_{g}^{2}\right)^{-1 / 2}$. One 
can see that the initial momentum of the trapped electron has a minimum, $p_{\min }$, corresponding to the minimum of the wake potential, $\Phi_{\min }$; at this trapping point the wake electric field $E_{z}=-\left(1 / \beta_{g}\right)^{2} \mathrm{~d} \Phi / \mathrm{d} \xi$ (normalized to $m_{e} c \omega_{p} / e$ ) is zero. The maximum value $p_{\max }=p_{g}$ corresponds to the point in the wake where $\Phi=1\left(E_{z}=-E_{z, \max }<0\right)$. In the nonlinear wakefield the longitudinal space interval, in which electrons can be trapped, decreases with the wake amplitude $E_{z, \text { nax }}$ (though the nonlinear wake wavelength increases) and is less than a quarter of the linear wake wavelength $\lambda_{p}=2 \pi / k_{p}$. Figure 2 shows numerical results for the minimum trapping momentum and the wakefield amplitude in dependence on the peak amplitude $a_{0}$ of a Gaussian laser pulse: $a=a_{0} \exp \left[-\left(\xi-\xi_{x}\right)^{2} / \sigma_{z}^{2}\right]$. As usual, the electric field amplitude $a$ of the pulse is normalized to $m_{e} c \omega_{L} / e$, where $\omega_{L}$ is the laser frequency. In this paper values of $a_{0}=2, \gamma_{s}=50, \sigma_{z}=2$, and $\xi_{c}=3 \sigma_{z}$ are used in the numerical calculations.

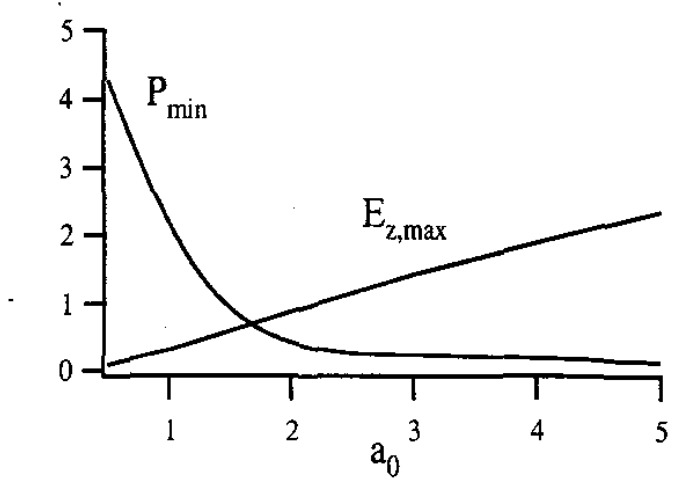

Figure 2. Calculated dependence of minimum initial momentum of trapped electrons, $p_{\min }$, and wakefield amplitude, $E_{z, \text { inax }}$, on the peak laser pulse amplitude, $a_{0}$.

For a moderately nonlinear wakefield with an amplitude much less than the wave-breaking field $\left[2\left(\gamma_{g}-1\right)\right]^{1 / 2}$, $\left|1-\Phi_{t r}\right|=\left|\phi_{r}\right|>>1 / \gamma_{g}<<1$. In this case, supposing that $p_{0}$ is not close to $p_{s}$, one can obtain from (1):

$$
p_{0} \approx\left(\phi_{t r}-1 / \phi_{r r}\right) / 2
$$

where $-1<\phi_{r}<0$. Then $p_{\min } \approx\left(\phi_{\min }-1 / \phi_{\min }\right) / 2$. In the linear wakefield $|\phi|<<1$, so $p_{\min } \approx 0.5 /\left|\phi_{\min }\right|>>1$. Thus, in the linear wakefield, only relativistic electrons can be trapped. However, for an initially relativistic electron, the trapping time increases proportional to $\gamma_{0}^{2}$.

The trapping time, $\tau_{t r}$, which is the time interval, that is necessary for an electron to reach the trapping point $\xi_{r}\left(p_{0}\right)$, depends on the initial momentum $p_{0}$ and on the initial position $\xi_{0}$ :

$$
\tau_{r r}\left(\xi_{0}, p_{0}\right)=\tau_{t r 0}\left(p_{0}\right)+\xi_{0} /\left(1-\beta_{0} / \beta_{g}\right)
$$

where $\tau_{t r 0}$ is the trapping time of an electron which is initially at $\xi=0$. The dependence $\tau_{r r}\left(p_{0}\right)$ is shown in Fig. 3 for $a_{0}=2$ and $\gamma_{g}=50$; in this case $E_{z, \max }=0.9$ (see Fig. 1), the nonlinear plasma wavelength is $A_{p} \approx 1.144 \lambda_{p}$, and $p_{\min }=0.53$. The trapping time has a minimum, which corresponds to $p_{0} \approx 1$, or to a kinetic energy of about 200 $\mathrm{keV}$. For the trapping time of an electron bunch, in which the electron momentum changes in the range $p_{1} \leq p_{0} \leq p_{2}$, and, supposing that the tail of the bunch is at $\xi=0$, we can write:

$$
\Delta \tau_{t r} \approx\left(\tau_{t r, \text { max }}-\tau_{t r, \text { min }}\right)+L_{0} /\left(1-\beta_{2} / \beta_{g}\right)
$$

where $\tau_{t r \text {,max }(\min )}$ is the maximum (minimum) value of $\tau_{t r 0}$ for electrons in the range $p_{1} \leq p_{0} \leq p_{2}$, and $L_{0}$ is the initial bunch length.

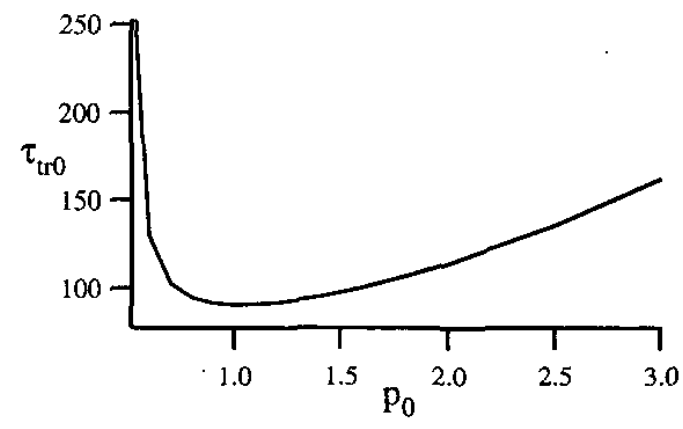

Figure 3 . The trapping time, $\tau_{t n}$, as a function of the initial electron momentum, $p_{0}$.

As the trapped electron has reached a relativistic energy $(y>>1)$ in the wakefield, its dynamics becomes slow, and its spatial position in the wake changes on a normalized time scale of $\tau_{a c c}=2 \pi \gamma_{g}^{2}$. So, another electron, with the same $p_{0}$ but different $\xi_{0}$ will be trapped very close to the first one if $\Delta \tau_{r r}<<\tau_{a c c}$. Test particle simulations showed that the trapped bunch length is much less than the plasma wavelength and undergoes practically no change during acceleration. One can see that the absolute and relative energy spread in the trapped bunch are $\delta \gamma \approx \Delta \tau_{r r}\left|E_{z}\left(\xi_{r}\right)\right|$ and $\varepsilon=\delta \gamma / \gamma \approx \Delta \tau_{r r} /\left(\tau-\Delta \tau_{r r}\right)$ respectively [4,5]. In the case of finite momentum spread $\delta_{0}$ in the initial bunch, electrons with different $p_{0}$ will be trapped at different trapping points. From Eq. (2) $\phi_{r} \approx p_{0}-\gamma_{0}$. Then, for an electron bunch $\delta \phi_{t r}=\phi_{t r}\left(p_{2}\right)-\phi_{t r}\left(p_{1}\right) \approx\left|E_{z}\right| L \approx \delta \gamma_{0}-\delta p_{0}$, where $E_{z}$ is some value of the wake electric field in the region occupied by the trapped bunch, $L$ is the trapped bunch length. So, the trapped bunch length can be estimated as $L \approx\left(\delta / \gamma_{0}-\delta p_{0}\right) /\left|E_{z}\right|$ when the effect of the finite initial bunch 
length is less than the effect of the initial momentum spread.

In Fig. 4 the energy of trapped electrons is shown for the case of an initially mono-energetic bunch (in this case the trapped bunch length and the absolute energy spread are approximately proportional to the initial bunch length), $p_{0}=1$, and $L_{0}=10 \lambda_{p}$. For example, at an acceleration length $l=400 \lambda_{p}$ (corresponding to $2 \mathrm{~cm}$ for $\lambda_{p}=50 \mu \mathrm{m}$ ), the trapped bunch length is about $7 \times 10^{-3} \lambda_{p}(\approx 0.35 \mu \mathrm{m}$ in the case of $\lambda_{p}=50, \mathrm{~mm}$ ) when $L_{0}=10 \lambda_{p}$ and 10 times less when $L_{0}=\lambda_{p}$; the trapped bunch length change is insignificant during acceleration. The energy of the electrons is about 1 $\mathrm{GeV}$ at $l=400 \lambda_{p}$, the relative energy spread is $\approx 0.93 \%$ for $L_{0}=\lambda_{p}$ and $\approx 9.5 \%$ when $L_{0}=10 \lambda_{p}$. The generation of good quality ultra-short relativistic e-bunches in the case of large initial momentum spread and $L_{0} \sim \lambda_{p}$ is also possible $[4,5]$. The initial bunch can be generated for example by a laser-driven photo-cathode RF gun.

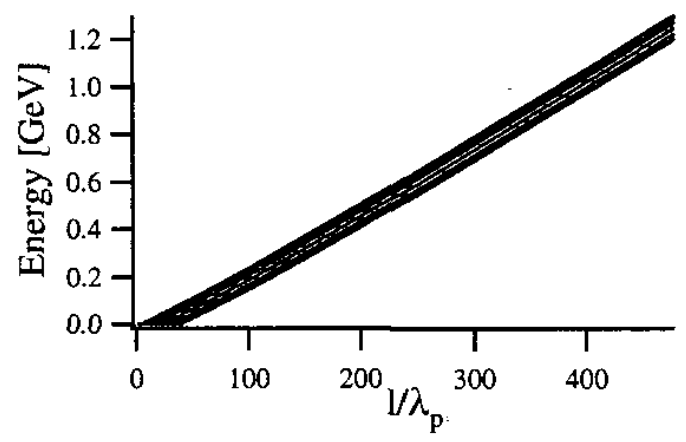

Figure 4. Energy of accelerating electrons, $p_{0}=1, L_{0}=10 \lambda_{p}$.

\section{THREE-DIMENSIONAL (3D) CASE}

In a 3D linear wakefield, generated in an uniform plasma, the transverse force is defocusing in the trapping region. Fortunately, in the nonlinear wakefield and in the wake generated in a plasma channel the near-axis wakefield can be focusing over the entire accelerating region $[5,6]$, so that our scheme can "work" in 3D as well. The trapped electrons are concentrated near the axis due to the focusing force. An example of the radial motion of the electrons with different initial radial positions is shown in Fig. 5 for the case of a laser wakefield excited in a plasma channel by a pulse with a Gaussian $\left(-\exp \left(-r^{2} / \sigma_{r}^{2}\right)\right)$ radial profile. For these initial bunch parameters and $L_{0}=\lambda_{p}$, the trapped bunch length is $L=3.5 \times 10^{-3} \lambda_{p}$, the relative energy spread is $\varepsilon \approx 3 \%$ at the acceleration distance $l=400 \lambda_{p}$, and $L \approx 0.01 \lambda_{p}$ and $\varepsilon \approx 10 \%$ when $L_{0}=10 \lambda_{p}$. In the case of finite momentum spread in the initial bunch, namely, $0.9 \leq p_{z 0} \leq 1.2,-0.02 \leq p_{r 0} \leq 0.02$ $\left(1.35 \leq \gamma_{0} \leq 1.56\right)$, and $L_{0}=0.8 \lambda_{p}$, the trapped bunch length is about $0.02 \lambda_{p}$ during acceleration. The average energy and the relative energy spread in the accelerated bunch are $\approx 1.07 \mathrm{GeV}$ and $\approx 4.5 \%$ respectively, at $l=400 \lambda_{p}$. The longitudinal dynamics and energy of the trapped electrons in $3 \mathrm{D}$ are approximately the same as in the $1 \mathrm{D}$ case.

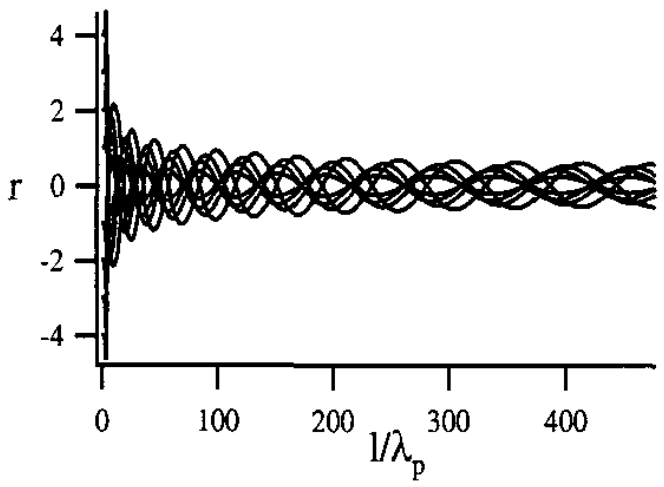

Figure 5. Transverse dynamics of trapped electrons, $p_{z 0}=1, p_{r 0}=0, a_{0}=2, \sigma_{z}=2, \sigma_{r}=5$.

In $3 \mathrm{D}$, the normalized emittance was calculated to be $\varepsilon_{n} \sim\left(R^{2} \Omega / 4 \pi^{2}\right) \lambda_{p}[5]$, where $\Omega=\left(\mid \partial f_{r} / \partial r / \gamma\right)^{1 / 2}$ is the betatron frequency, $R<<\sigma_{r}$ is the trapped bunch radius ( $\Omega$ and $R$ are in the normalized units), and is in the order of a nanometer in the ultra-relativistic regime. For example, $\varepsilon_{n}-3 \mathrm{~nm}$ for the case presented in Fig. 5 (initially monoenergetic bunch) when $L_{0}=10 \lambda_{p}, l=400 \lambda_{p}$ and $\lambda_{p}=50 \mu \mathrm{m}$; in the case of the finite initial momentum spread considered above, the emittance is approximately the same.

The total number of accelerated electrons is restricted by the beam loading effect, $\mathrm{N}_{t o t}<<3 \times 10^{7} \lambda_{p}[\mu \mathrm{m}]$ [5]. One can see that this number can reach a value of $\sim 10^{8}$ when the plasma wavelength is in the order of tens of microns (that is typical for laser pulses with $\lambda_{L} \sim 1 \mu \mathrm{m}$ ) and $\sim 10^{9}$ for $\lambda_{p}$ in order of hundreds microns (that is typical for $\mathrm{CO}_{2}$ laser pulses, $\lambda_{L} \approx 10 \mu \mathrm{m}$ ). The scheme can also be applied to the self-modulated LWFA case.

\section{REFERENCES}

[1] X. J. Wang, X. Qiu, and I. Ben-Zvi, Phys. Rev. E 54, R3121 (1996).

[2] P. Kung, H.-C. Lihn, H. Wiedemann, and D. Bocek, Phys. Rev. Lett. 73, 967 (1994).

[3] B. E. Carlsten and S. J. Russell, Phys. Rev. E 53, R2072 (1996).

[4] A. G. Khachatryan, JETP Letters 74, 371 (2001).

[5] A. G. Khachatryan, Phys. Rev. E 65, 046504 (2002).

[6] N. E. Andreev, L. M. Gorbunov, V. I. Kirsanov, K. Nakajima, and A. Ogata, Phys. Plasmas 4, 1145 (1997). 